\title{
Social determinants of unhealthy lifestyles
}

The references in this letter (BMJ 2018;363:k4349, doi:10.1136/ bmj.k4349) were misnumbered. They have been corrected in the online version. 